ARTICLE

Received 28 Jan 2014 | Accepted 30 Jul 2014 | Published 10 Sep 2014 DOl: 10.1038/ncomms5863

\title{
Spontaneous transmembrane helix insertion thermodynamically mimics translocon-guided insertion
}

\author{
Martin B. Ulmschneider ${ }^{1}$, Jakob P. Ulmschneider ${ }^{2}$, Nina Schiller ${ }^{3,4}$, B.A. Wallace ${ }^{5}$, Gunnar von Heijne ${ }^{3,4}$
} \& Stephen H. White ${ }^{6}$

The favourable transfer free energy for a transmembrane (TM) $\alpha$-helix between the aqueous phase and lipid bilayer underlies the stability of membrane proteins. However, the connection between the energetics and process of membrane protein assembly by the Sec61/SecY translocon complex in vivo is not clear. Here, we directly determine the partitioning free energies of a family of designed peptides using three independent approaches: an experimental microsomal Sec61 translocon assay, a biophysical (spectroscopic) characterization of peptide insertion into hydrated planar lipid bilayer arrays, and an unbiased atomic-detail equilibrium folding-partitioning molecular dynamics simulation. Remarkably, the measured free energies of insertion are quantitatively similar for all three approaches. The molecular dynamics simulations show that TM helix insertion involves equilibrium with the membrane interface, suggesting that the interface may play a role in translocon-guided insertion.

\footnotetext{
${ }^{1}$ Department of Materials Science and Engineering, Johns Hopkins University, Baltimore, Massachusetts 21218, USA. ${ }^{2}$ Institute of Natural Sciences and Department of Physics and Astronomy, Shanghai Jiao Tong University, Shanghai 200240, China. ${ }^{3}$ Center for Biomembrane Research, Department of Biochemistry and Biophysics, Stockholm University, SE-106 91 Stockholm, Sweden. ${ }^{4}$ Science for Life Laboratory, Stockholm University, SE-17177 Solna, Sweden. ${ }^{5}$ Institute of Structural and Molecular Biology, Birkbeck College, University of London, Malet Street, London WC1E 7HX, UK. ${ }^{6}$ Department of Physiology \& Biophysics and the Center for Biomembrane Systems, University of California, Irvine, California 92697-4560, USA. Correspondence and requests for materials should be addressed to M.B.U. (email: martin@ulmschneider.com) or to S.H.W. (email: stephen.white@uci.edu).
} 
T ransmembrane (TM) $\alpha$-helices of proteins embedded in plasma membranes are highly enriched in amino acids with non-polar side chains, such as leucine and isoleucine. Potential TM helices in an amino-acid sequence can be identified using hydropathy plots ${ }^{1,2}$, which are based upon the slidingwindow method ${ }^{3}$. If a true thermodynamic hydrophobicity scale is used ${ }^{4}$ with a sliding window of about 20 amino acids, the window sum is an estimate of the free energy of transfer of an $\alpha$-helix between the aqueous phase and the 30-Å-thick hydrocarbon core of the lipid bilayer $\left(\Delta G_{\mathrm{wbi}}\right.$, Fig. 1a). Although this free energy is clearly relevant to the question of TM helix stability, its connection to the energetics of TM helix insertion in vivo by the Sec translocon $\left(\Delta G_{\text {app }}\right.$, Fig. 1a) is far from clear. Understanding the connection between $\Delta G_{\mathrm{wbi}}$ and $\Delta G_{\mathrm{app}}$, which is the goal of the work presented here, is crucial for a quantitative description of membrane protein assembly, folding, and stability.

Membrane proteins are assembled with the aid of the heterotrimeric Sec translocon, specifically Sec $61 \alpha \beta \gamma$ in mammals and SecYEG in bacteria ${ }^{5-8}$. The SecY/Sec61 complex also guides the secretion of soluble proteins. Insertion and secretion of polypeptides occur cotranslationally in eukaryotes, as the nascent chain emerges from translocon-docked ribosomes. The Sec61 complex acts as a switch that directs sufficiently hydrophobic segments of elongating nascent chains into the membrane as TM helices while allowing more polar secreted segments to pass through the membrane into the interior of the endoplasmic reticulum (ER). The TM selection rules followed by the Sec61 complex have been determined ${ }^{9,10}$ by means of an in vitro expression system supplemented with dog pancreas ER vesicles (rough microsomes) using hundreds of so-called $\mathrm{H}$-segments inserted into a host protein, Escherichia coli leader peptidase ${ }^{11}$. The rules are described by means of apparent free energies of transfer $\left(\Delta G_{\text {app }}\right)$ of helices between the translocon and bilayer (Fig. 1).

Establishing a quantitative relationship between $\Delta G_{\text {app }}$ and the water-to-bilayer free energy of transfer is problematic, because direct experimental determinations of $\Delta G_{\mathrm{wbi}}$ are rarely possible owing to aggregation of highly hydrophobic peptides in the aqueous phase ${ }^{12}$. Direct determinations are theoretically possible at aqueous concentrations low enough to prevent aggregation, but the concentrations required are so low as to be impractical to measure accurately in most cases. The only practical approach is to use microsecond-scale equilibrium molecular dynamics simulations, which can measure, in essence, water-to-bilayer partitioning at infinite dilution. Using this approach, Ulmschneider et al. ${ }^{13}$ measured the partitioning of ace- $\mathrm{L}_{n-}$ amide and ace-GGPG- $\mathrm{L}_{n}$-GPGG-amide peptides $(n=5 \quad \ldots \quad 12)$ into palmitoyloleoylphosphatidycholine (POPC) bilayers and compared the resulting $\Delta G_{\text {wbi }}$ free energies with experimental measurements ${ }^{14}$ of $\Delta G_{\text {app }}$. Two important observations resulted. First, consistent with the very low aqueous solubility of hydrophobic peptides, the simulations revealed that the peptides quickly adsorbed at the membrane interface and never subsequently desorbed. The measured values of $\Delta G_{\text {wbi }}$ thus corresponded to transfer free energies from the bilayer interface to TM orientations across the bilayer hydrocarbon core. This observation is completely consistent with extensive studies of the interfacial partitioning of peptides ${ }^{2}$. The second important observation was that the $\Delta G_{\text {wbi }}(n)$ values exactly paralleled the $\Delta G_{\text {app }}(n)$ values with a simple offset of about $-2 \mathrm{kcal} \mathrm{mol}^{-1}$ with $\Delta G_{\text {wbi }}(n)$.

A fundamental question is how the observed offset would be affected by changing the polarity of the $\mathrm{H}$-segment. To begin answering that question, we have altered the polarity of the segment by introducing a single arginine at the center of the polyleucine H-segment. We chose arginine for three reasons. First, the introduction of a charged residue should cause a strong perturbation of $\mathrm{H}$-segment polarity. Second, Arg cannot be deprotonated when inserted into the center of a lipid bilayer based upon nuclear magnetic resonance measurements ${ }^{15}$ and simulations ${ }^{16}$; this eliminates complications that might arise from protonation-deprotonation reactions. Third, the use of Arg provided an opportunity to examine further the contentious finding of Hessa et al. ${ }^{10}$ that the free-energy cost $\Delta G_{\text {app }}^{\text {Arg }}$ of inserting a single arginine into the center of a TM helix is only $+2 \mathrm{kcal} \mathrm{mol}^{-1}$.

This small value of $\Delta G_{\text {app }}^{\text {Arg }}$ was contentious, because computational models predicted prohibitively large penalties of

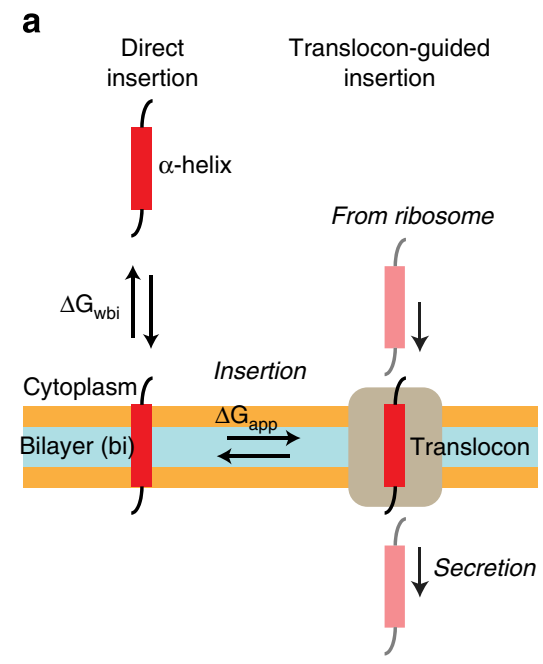

\section{b}

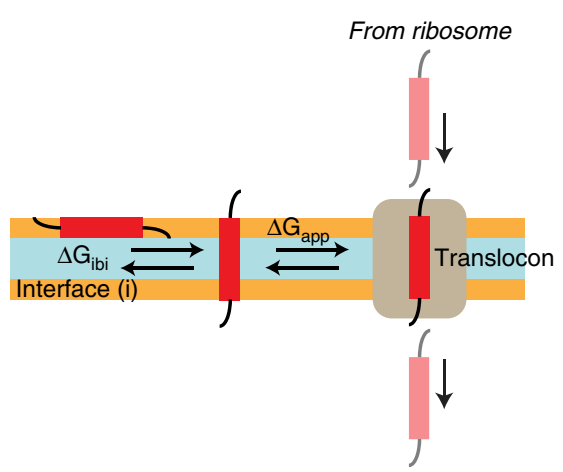

Figure 1 | Schematic comparisons of spontaneous transmembrane (TM) helix insertion with translocon-guided insertion. (a) The thermodynamic stability of TM helices is generally thought of as the free energy of transfer $\Delta G_{w b i}$ of a helix from water to bilayer. Translocon-guided insertion of helices is characterized by an apparent free energy of transfer $\Delta G_{\text {app }}$ from translocon to bilayer. The fundamental question addressed in this paper is the relationship between the two free-energy values. (b) All-atom equilibrium simulations presented in this paper show that helical peptides are either across the membrane (TM) or on the membrane interfaces, indicating that the actual free energy determined by the simulations $\Delta G_{\text {ibi }}$ is for transfer from the bilayer interface to TM. This figure is based on Fig. 7 of Schow et al. ${ }^{24}$ 
$15 \mathrm{kcal} \mathrm{mol}^{-1}$ or more ${ }^{17-26}$. Much of the disagreement has been due to disconnection between the quantities that the simulations and experiments actually measure. While the experimental assay measured the propensity with which the mammalian Sec61 translocon inserts 19-residue arginine-containing TM segments into the ER membrane, the computational methods calculated the free energy of burying an isolated guanidinium group in the hydrophobic core of a lipid bilayer. Attempts have been made to connect the two results via thermodynamic cycles ${ }^{24-27}$. However, accurate determination of the free-energy components of a thermodynamic cycle depends crucially on a detailed knowledge of the structural and conformational ensemble of the equilibrium states, between which the free energies are to be determined. For the translocon assay, these states are currently not known $9,10,26$, while the computational free-energy calculations are based on empirical assumptions about the nature of these states.

In the experiments reported here, we used three independent approaches to examine polyleucine segments carrying a central Arg residue: (a) an experimental microsomal Sec61 translocon assay, (b) spectroscopic characterization of peptide insertion into hydrated planar lipid bilayer arrays, and (c) an unbiased atomicdetail equilibrium folding-partitioning molecular dynamics simulation. Great care was taken to design the study such that all three methods could be compared directly with each other.

Direct quantification, both experimentally and computationally, of the free-energy difference $(\Delta G)$ between two states requires that both states be measurably populated at equilibrium. In the present context, this is best achieved by designing TM segments that have partitioning free energies close to $\Delta G=0$, so that the populations of both the inserted and non-inserted states are about equal. Because the $\Delta G$ of a peptide can only be estimated at the design stage, we chose a series of symmetric polyleucine peptides of the type $\mathrm{G}-\mathrm{L}_{n} \mathrm{RL}_{n}-\mathrm{G}(n=5,6,7,8)$. Varying the number of flanking leucine residues on either side of the central arginine allowed the partitioning free energy to be modulated systematically, with longer peptides expected to insert more favourably into the lipid bilayer than shorter segments, which cannot fully span the bilayer. This allowed selection of peptides for which $\Delta G$ could be determined accurately from the inserted- and non-inserted-state populations. A polyleucine scaffold was chosen, because it forms near-perfect $\alpha$-helices, thus minimizing the risk of guanidinium side chain interactions with the peptide backbone. The peptide termini were acetylated ( $\mathrm{N}$ terminus) and amidated ( $\mathrm{C}$ terminus) to avoid kinetic barriers that arise from having to transport charged end-groups across the hydrophobic bilayer core.

\section{Results}

$\Delta \boldsymbol{G}$ from the translocon assay. The insertion propensity of the peptides into the ER membrane was determined using a microsomal translocation assay ${ }^{9,10}$. Each peptide sequence was introduced as an extra $\mathrm{H}$-segment into the large luminal P2 domain of the model protein leader peptidase (LepB) from E. coli. Each H-segment was flanked by GGPG- and -GPGG tetrapeptides at its $\mathrm{N}$ - and $\mathrm{C}$ termini, respectively, to 'insulate' the $\mathrm{H}$-segment from the LepB carrier protein ${ }^{9,10}$. Glycosylation sites were placed on either side of the segment to allow quantitation of the insertion propensity. The protein was then expressed in vitro in the presence of ER-derived dog pancreas rough microsomes. Because glycosylation only takes place in the interior of the microsome, membrane-inserted segments are singly glycosylated, whereas translocated segments are doubly glycosylated. The insertion propensity $P_{\mathrm{TM}}=f_{1 \mathrm{~g}} /\left(f_{1 \mathrm{~g}}+f_{2 \mathrm{~g}}\right)$ of the segment was determined by phosphoimager scans of SDSpolyacrylamide gel electrophoresis (PAGE) gels that measure the fractions of singly $\left(f_{1 \mathrm{~g}}\right)$ and doubly $\left(f_{2 \mathrm{~g}}\right)$ glycosylated proteins. As shown in Fig. 2a and Supplementary Fig. 1, the SDS-PAGE results reveal that increasing the number of leucine residues shifts the insertion propensity from a predominantly non-inserted configuration (for $\mathrm{L}_{5} \mathrm{RL}_{5}$ ) towards a fully inserted configuration (for $\mathrm{L}_{8} \mathrm{RL}_{8}$ ).

$\Delta G$ from synthetic peptides in oriented lipid bilayers. To analyse insertion of the corresponding synthetic $\mathrm{L}_{n} \mathrm{RL}_{n}$ peptides, the time-averaged orientation of the peptides in oriented planar lipid bilayer arrays was determined using oriented synchrotron radiation circular dichroism (OSRCD) spectroscopy. All four peptides were synthesized using solid-phase synthesis and purified using reverse-phase high-pressure liquid chromatography (see Supplementary Information). The sequences differed from the equivalent $\mathrm{H}$-segments in that the GGPG- and -GPGG insulating flanks were replaced by a single glycine, which avoids dilution of the helical circular dichroism spectrum of the $\mathrm{L}_{n} \mathrm{RL}_{n}$ segment by the unstructured flanks (see Methods). To facilitate purification and concentration measurements, the N-terminal leucine of each peptide was replaced by a tryptophan, which has the same $\Delta G_{\text {app }}$ as leucine in the translocon assay when near the ends of the TM helices. The validity of this approach was verified by control simulations of the $\mathrm{GWL}_{6} \mathrm{RL}_{7} \mathrm{G}$ peptide, which showed the insertion probability to be identical to that of the $\mathrm{GL}_{7} \mathrm{RL}_{7} \mathrm{G}$ peptide (see Supplementary Fig. 2; Table 1). Oriented multibilayer arrays were formed by depositing an organic solution containing both peptide and lipid onto quartz substrates. Highly oriented arrays form spontaneously after slow evaporation of the organic solvent followed by vapor-phase hydration at $100 \%$ relative humidity ${ }^{12,28,29}$. Films formed in this way typically contain stacks of $\sim 1,000$ bilayers highly aligned parallel to the quartz substrate, as determined by neutron diffraction ${ }^{30}$

The net orientation of the synthetic peptides inside the planar POPC bilayer stacks was determined by recording SRCD spectra with the light beam perpendicular to the quartz substrate ${ }^{31,32}$. Figure $2 \mathrm{~b}$ shows the measured spectra together with the theoretically predicted spectra for $\alpha$-helices aligned perpendicular and parallel to the bilayer normal. Spectra could only be obtained for two peptides: the spectrum of $L_{8} R_{8}$ is typical for a TM helix, while the spectra for $\mathrm{L}_{7} \mathrm{RL}_{7}$ and $\mathrm{L}_{6} \mathrm{RL}_{6}$ suggest that the conformational population averages contain an increasing proportion of surface-bound helical conformers. For $\mathrm{L}_{6} \mathrm{RL}_{6}, P_{\mathrm{TM}} \approx 30-50 \%, \Delta G \approx 0-0.5 \mathrm{kcal} \mathrm{mol}^{-1}$ ) and for $\mathrm{L}_{7} \mathrm{RL}_{7}$ $P_{\mathrm{TM}} \approx 70-90 \%, \Delta G \approx-0.5$ to $-1.1 \mathrm{kcal} \mathrm{mol}^{-1}$. The spectrum for $\mathrm{L}_{5} \mathrm{RL}_{5}$ is atypical, with peaks at $229 \mathrm{~nm}$ indicative of interfacial aggregates (Supplementary Fig. 3).

$\Delta \boldsymbol{G}$ from folding-partitioning simulations. Finally, to determine computationally the free energy of peptide insertion into the membrane of glycine-flanked segments (see Methods), we used unbiased multi-microsecond atomic-detail folding-partitioning simulations at an elevated temperature of $140^{\circ} \mathrm{C}$. We have recently demonstrated that this in silico approach allows direct quantification of the transfer free energy of thermostable peptides from the equilibrium ensembles of peptide configurations in the presence of a lipid bilayer ${ }^{13,33,34}$. As in the synthetic implementation, the GGPG- and -GPGG insulating flanks were replaced by a single glycine, to make them identical to the synthetic implementation and to speed up partitioning kinetics, because transporting the unstructured GGPG/GPGG flanks across the hydrophobic bilayer core during TM insertion of the peptide is associated with a large energetic penalty. The removal of the flanking segments has been shown not to affect the overall 
a

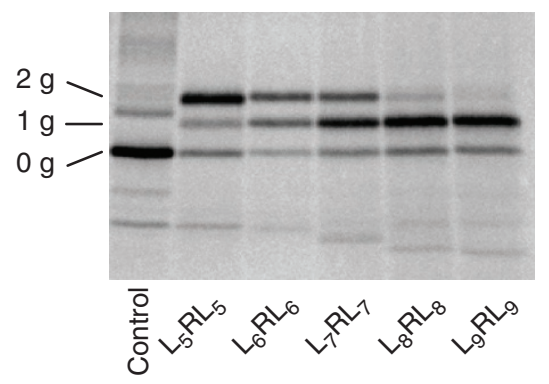

C

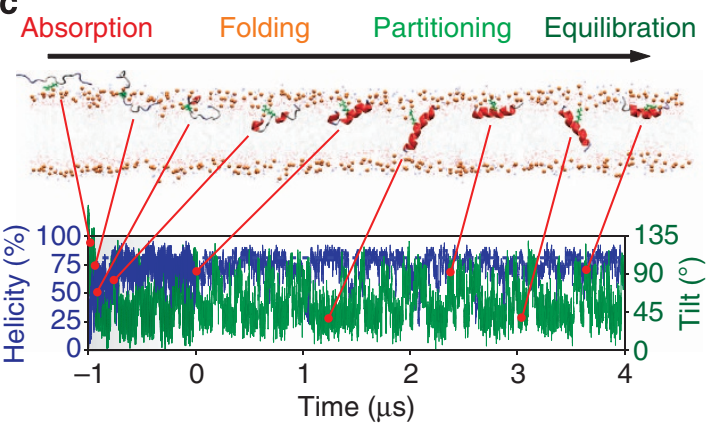

b

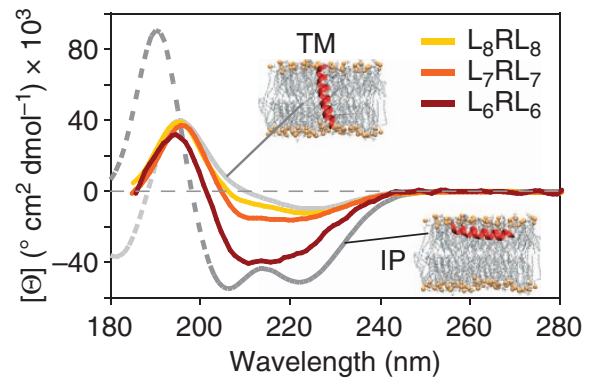

d

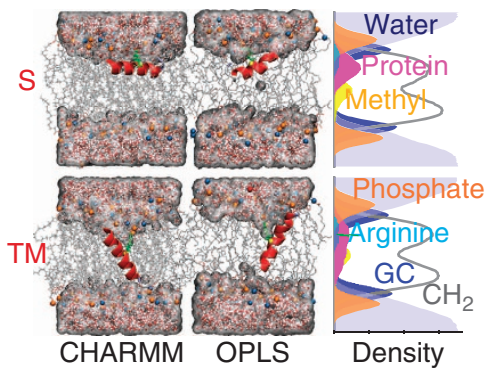

Figure 2 | Experimental data for in vitro translocon-guided insertion, orientation of synthetic peptides in POPC lipid bilayers and simulations of spontaneous insertion. (a) SDS-PAGE analysis of LepB constructs containing the GGPG- $\mathrm{L}_{n} R L_{n}-\mathrm{GPGG}$ peptides as an added $\mathrm{H}$-segment. Glycosylation sites on either side of the segment allow quantification of inserted (singly glycosylated, $1 \mathrm{~g}$ ) and non-inserted (doubly glycosylated, $2 \mathrm{~g}$ ) configurations. Increasing the number of leucine residues in the $\mathrm{H}$-segment shifts the equilibrium from doubly to singly glycosylated peptides, with $\mathrm{L}_{6} \mathrm{RL}_{6}$ and $\mathrm{L}_{7} \mathrm{R} \mathrm{L}_{7}$ showing substantial populations of both forms. Proteins that remain unglycosylated $(0 \mathrm{~g})$, never entered the translocon and are excluded from the measurement. The full SDS-PAGE gel is presented in Supplementary Fig. 1. (b) Oriented synchrotron radiation circular dichroism (OSRCD) spectra of the G-WL $-L_{n-1} R L_{n}-G$ peptides in stacked POPC lipid bilayers (peptide/lipid $=1 / 100$, relative hydration $=100 \%$ ). Theoretical reference spectra ${ }^{31,32}$ for $\alpha$-helical peptides oriented parallel $\left(T M\right.$, tilt angle $\left.\approx 0^{\circ}\right)$ and perpendicular (IP, tilt angle $\approx 90^{\circ}$ ) to the membrane normal (coaxial to the light beam) are shown in grey. (c) Unbiased folding-partitioning simulation of the $G-L_{7} R L_{7}-G\left(L_{7} R L_{7}\right)$ peptide (c.f. Table 1). Absorption and interfacial folding is rapid and typically completed within $400 \mathrm{~ns}$. The arginine residue, which is highlighted in green, retains contact with the lipid headgroup phosphates at all times (see Supplementary Table 1). (d) Representative snapshots of the $L_{7} R L_{7}$ peptide simulation showing the two dominant states present at equilibrium. The configurations consist of a surface (S)-adsorbed and a transmembrane (TM)-inserted helical peptide, irrespective of the force field employed. The density profiles are averages over the $S$ and TM states of the OPLS simulation of $L_{7} R L_{7}\left(t_{S}=70 \mathrm{~ns}, t_{T M}=130 \mathrm{~ns}\right)$. GC indicates the glycerol backbone distribution. In the TM state, the arginine residue is buried more deeply in the hydrophobic region of the bilayer. Phosphates (orange spheres) and water are frequently pulled into the bilayer to help solvate the charged guanidinium group. The Gaussian distribution of the peptide on the bilayer leaflet is noticeably broader and reaches deeper into the hydrophobic bilayer core.

Table 1 | Summary of simulation results for polyleucine-based peptides in POPC lipid bilayers.

\begin{tabular}{|c|c|c|c|c|c|c|c|c|}
\hline Peptide sequence & Length $(\mu \mathrm{s})$ & $\mathbf{T}\left({ }^{\circ} \mathbf{C}\right)$ & Helicity (\%) & $\boldsymbol{N}_{\text {trans }}$ & $<\mathbf{t}>_{\text {TM }^{\star}}{ }^{\star}$ (ns) & $<\mathbf{t}>\mathbf{s}^{\star}$ (ns) & $P_{\mathrm{TM}}(\%)$ & $\Delta G_{\mathrm{S} \rightarrow \mathrm{TM}}\left(\mathrm{kcal} \mathrm{mol}^{-1}\right)$ \\
\hline $\mathrm{GL}_{5} \mathrm{RL}_{5} \mathrm{G}$ & 4 & 140 & $64 \pm 23$ & 146 & $4 \pm 3$ & $49 \pm 56$ & $7.0 \pm 1.6$ & $2.1 \pm 0.2$ \\
\hline $\mathrm{GL}_{7} \mathrm{RL}_{7} \mathrm{G}$ & $1+4$ & 140 & $77 \pm 13$ & 262 & $20 \pm 1$ & $11 \pm 1$ & $64.2 \pm 5.3$ & $-0.5 \pm 0.2$ \\
\hline $\mathrm{GL}_{8} \mathrm{RL}_{8} \mathrm{G}$ & 4 & 140 & $79 \pm 10$ & 106 & $63 \pm 73$ & $10 \pm 24$ & $86.2 \pm 4.7$ & $-1.5 \pm 0.3$ \\
\hline $\mathrm{GL}_{6} \mathrm{RL}_{6} \mathrm{G}$ & 6 & 90 & $68 \pm 13$ & 65 & $37 \pm 65$ & $125 \pm 165$ & $29.9 \pm 24.4$ & $0.8 \pm 1.4$ \\
\hline $2 \times \mathrm{GL}_{6} \mathrm{RL}_{6} \mathrm{G} \ddagger$ & 4 & 140 & $70 \pm 13$ & $170 \pm 29$ & $14 \pm 17$ & $33 \pm 39$ & $30.2 \pm 7.9$ & $0.7 \pm 0.4$ \\
\hline $\mathrm{GWL}_{6} \mathrm{RL}_{7} \mathrm{G}$ & 4 & 140 & $83 \pm 8$ & 163 & $32 \pm 34$ & $16 \pm 24$ & $65.8 \pm 9.1$ & $-0.5 \pm 0.4$ \\
\hline
\end{tabular}

$\Delta G_{S \rightarrow T M}$, insertion free energy; $N_{\text {trans, }}$ number of insertion/exit events; $P_{\mathrm{TM}}$, propensity of the TM state; $\mathrm{T}$, temperature; $\left.<t\right\rangle_{\mathrm{S}}$, average lifetime of the $\mathrm{S}$ state; $<t>\mathrm{TM}$, average lifetime of the $\mathrm{TM}$ state; TM, transmembrane.

The total simulation time was $35 \mu$ s. See Methods and Supplementary Information for details.

${ }^{\star}$ All averages are over the last $3.6 \mu$ s of the equilibrium phase of the simulations (that is, excluding the initial folding stage, which spanned the range $50-350$ ns).

†Control simulation with the CHARMM force field (CHARMM27 protein, CHARMM36 lipids, and TIP3P water). All other simulations were carried out with the OPLS force field (OPLS-AA protein, OPLS-UA lipids, and TIP3P water).

†Control simulation with two peptides.

partitioning free energies for a related series of polyleucine peptides $^{13}$.

At the beginning of each simulation, a single peptide was placed in bulk water about $15 \AA$ from the surface of a POPC lipid bilayer in a completely extended configuration. The peptide was then allowed to fold freely, as well as partition spontaneously into and out of the lipid bilayer. No restraints or biasing potentials were applied. Simulation timescales of $<400$ ns were sufficient to 
capture the initial non-equilibrium bilayer absorption and folding of the peptide, while a full characterization of the structural and configurational ensemble present at equilibrium typically required several microseconds. Figure $2 \mathrm{c}$ summarizes this method. The non-equilibrium part of the simulations is much shorter than for globular proteins of similar size. This is because the cost of a single unpaired backbone hydrogen-bonding group in the bilayer is very large ${ }^{2}\left(\approx 5 \mathrm{kcal} \mathrm{mol}^{-1}\right)$, which drives rapid secondary structure formation.

Table 1 summarizes the thermodynamic and kinetic quantities observed from the simulations. All peptides show only two states at equilibrium, with the first state ( $S$ ) consisting of a surface absorbed, predominantly helical peptide tilted at an angle of $\sim 90^{\circ}$ with respect to the membrane normal, and the second state (TM) consisting of a TM-inserted helix with a tilt of $\sim 30^{\circ}$ (Fig. 3). Each state is represented by a configurational and conformational ensemble (Supplementary Fig. 4), which is dominated by helical conformers (Supplementary Fig. 5). The bilayer responds to burial of the charged arginine side chain by localized deformation (Supplementary Fig. 6), caused by tight hydrogen-bonding interactions that pull down lipid phosphate headgroups into the bilayer core (Supplementary Table 1). This enables the guanidinium group to remain in contact with water even when deeply buried (Supplementary Fig. 7). No bulk-phase aqueous states are sampled at equilibrium, consistent with the experimentally confirmed insolubility of the peptides. That is, only two states are observed (see Fig. 4a): interface (S) and TM.

The partitioning equilibrium can be summarized in a peptidetilt versus insertion-depth free-energy surface (Supplementary Fig. 8). The data confirm that the equilibrium is between surfacebound and TM configurations for all peptides. Separation of the configurational ensemble into equilibrium states can be achieved by tracking the motion of the peptide termini along the membrane normal over the course of the simulation. Figure 3 demonstrates that this approach separates a TM configuration (where each bilayer interface hosts exactly one peptide terminus) from a surface-bound one (where one interface harbours both termini). Apart from allowing an accurate quantification of the S- and TM-state populations, this analysis allows calculation of kinetic properties such as the number of transitions and the average lifetimes of the states. All thermodynamic and kinetic data derived from Fig. 3 are summarized in Table 1, revealing a large number of equilibrium transitions between the $\mathrm{S}$ and TM states for all peptides.

Configurational equilibria in molecular dynamics simulations rely crucially on accurate force field parameters. To exclude a force field dependency of the results, the $\mathrm{L}_{7} \mathrm{RL}_{7}$ peptide simulation was repeated using the CHARMM force field (see Methods). This simulation showed a slightly more-helical and less-flexible peptide (Supplementary Fig. 9). The kinetics were sevenfold slower than for the equivalent OPLS force field simulation, but resulted in a very similar insertion free energy (Table 1). The difference in kinetics between the OPLS and CHARMM simulations is most likely due to the united-atom (OPLS) versus all-atom (CHARMM) nature of the lipid force fields ${ }^{35,36}$; the protein force field was all-atom in both cases.

For accurate determination of thermodynamic and kinetic quantities from folding-partitioning simulations, all thermally accessible states of the system must be fully sampled. Furthermore, great care must be taken to check that the resulting thermodynamic ensemble is fully converged and that the relative populations of the states present at equilibriums have stabilized at their equilibrium values. Tracking the running average of the TM-state propensity $\left(P_{\mathrm{TM}}\right)$ shows that at least $3 \mu$ s are required for convergence (Fig. 3c). Population averages derived from a

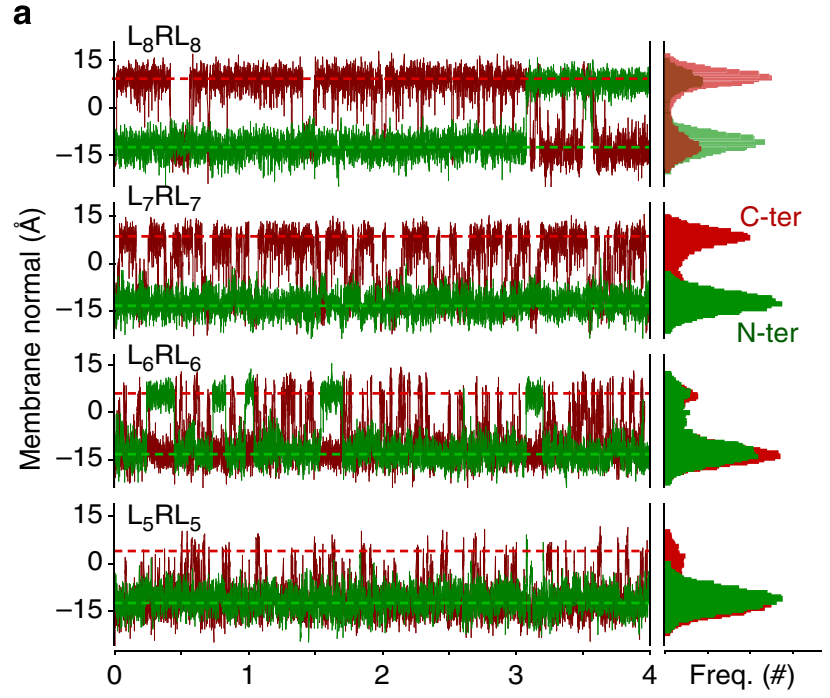

b

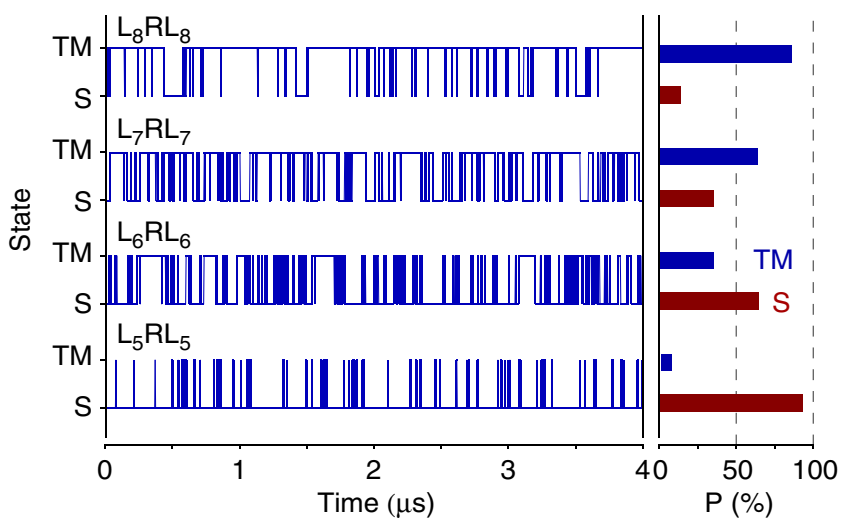

c

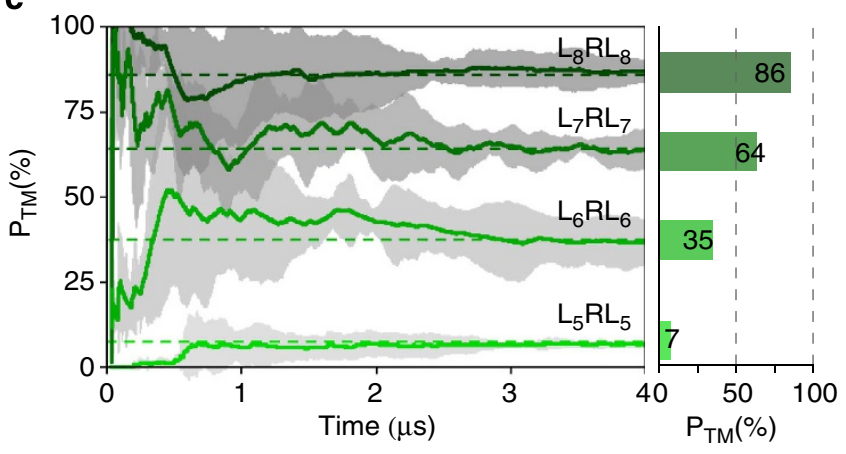

Figure 3 | Quantification of the kinetics and thermodynamics of peptide partitioning derived from equilibrium simulations. (a) Analysis of the positions of the peptide $\mathrm{N}$ termini (green) and $\mathrm{C}$ termini (red) along the membrane normal reveals the two-state behaviour of the system.

(b) Peptides in the transmembrane (TM) state have one terminus at each of the two membrane interfaces, while peptides in the surface-bound (S) state have both termini in the same membrane interface. This allows plotting the state transitions from the analysis of the peptide termini position along the membrane normal (a). Knowledge of the state as a function of simulation time allows direct determination of the $S$ and TM population averages, as well as analysis of state lifetimes, transition rates and related kinetics. The populations and associated free energies are given in Table 1. (c) Plots of the block-averaged TM-state propensity against the simulation time shows that the systems require at least $3 \mu$ s for full convergence. The equivalent kinetics for the $\mathrm{L}_{7} \mathrm{RL}_{7} \mathrm{CHARMM}$ simulation shows similar $\Delta G$, but much slower kinetics (see Supplementary Fig. 5). 
a
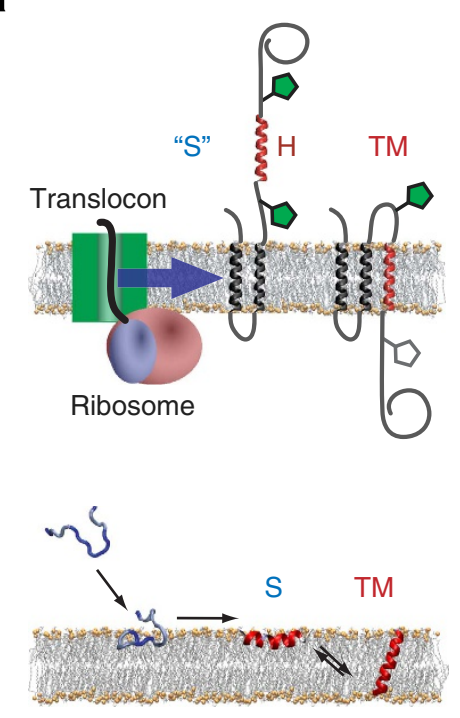

C

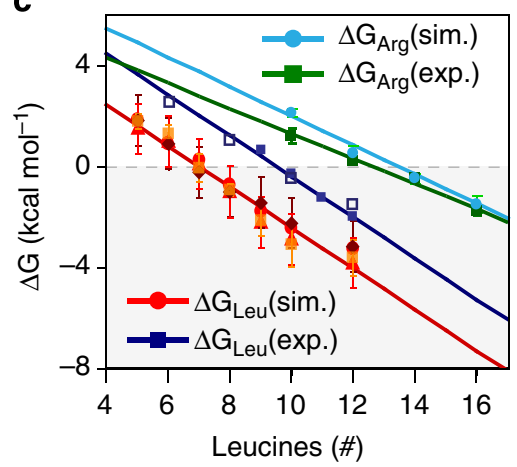

b
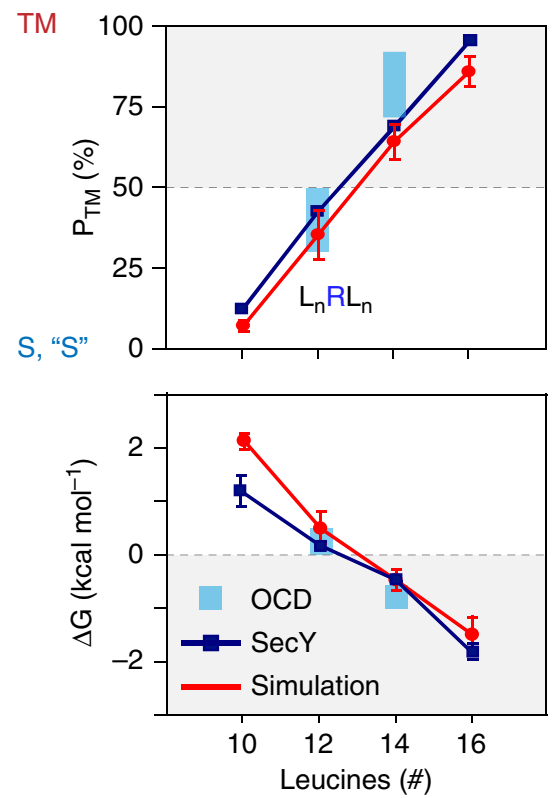

d

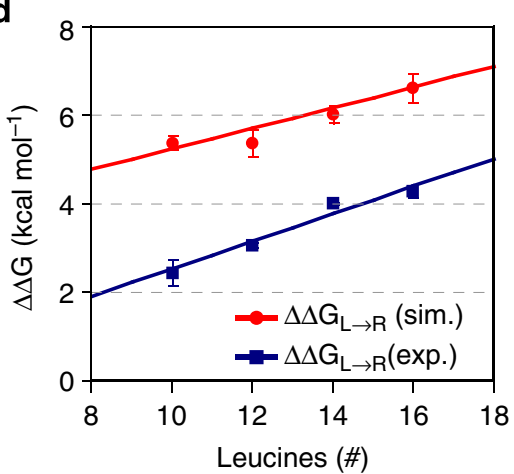

Figure 4 | Summary of insertion LnRLn propensity data and comparison with $\mathbf{L} \mathbf{2 n}$ data. The error bars are given by $\pm \sigma$, where $\sigma$ is the s.d. of the mean. (a) Schematic comparison of translocon-guided insertion (upper panel) with simulated spontaneous partitioning (lower panel). (b) Whole-peptide partitioning propensity $\left(P_{\mathrm{TM}}\right)$ and insertion free energy $(\Delta G)$ as a function of the total number of leucines of the $L_{n} R L_{n}$ peptides. Data in red circles show the results from the monomeric partitioning simulations (cf. Table 1). The blue squares show the experimental apparent insertion propensities measured from the Sec61 translocon assay, and the light blue data points show the results from the oriented circular dichroism (OCD) experiments, which could only be obtained for two peptides: $\mathrm{L}_{6} \mathrm{RL}_{6}\left(\mathrm{P}_{\mathrm{TM}} \approx 30-50 \%, \Delta \mathrm{G} \approx 0-0.5 \mathrm{kcal} \mathrm{mol}^{-1}\right)$ and $\mathrm{L}_{7} \mathrm{RL}_{7}\left(\mathrm{P}_{\mathrm{TM}} \approx 70-90 \%, \Delta G \approx-0.5\right.$ to $\left.-1.1 \mathrm{kcal} \mathrm{mol}{ }^{-1}\right)$. The structure of the singly glycosylated species is a TM helix, the structure and configuration of the doubly glycosylated peptide variant (corresponding to the $\mathrm{S}$ state) is not known, and is thus denoted by "S". (c) Comparison of polyleucine data with $\mathrm{L}_{n} R \mathrm{~L}_{n}$ data. The previously determined free energy of peptide insertion $\Delta G$ as a function of the total number of leucine residues shows a $\sim 2 \mathrm{kcal} \mathrm{mol}^{-1}$ offset between translocon experimental values (blue) and simulation-derived values (red) for pure polyleucine sequences ${ }^{13,14}$. However, for polyleucine sequences carrying a central arginine, the transloconbased experimental free energies (green) and simulation-derived partitioning free energies (light blue) are almost identical. (d) The free-energy penalty of replacing the central leucine with an arginine residue $\left(\Delta \Delta G_{L \rightarrow R}\right)$ is calculated by subtracting the $\Delta G$ of the equivalent polyleucine $\left(L_{n}\right)$ peptide from the $\Delta G$ of the $L_{n} R L_{n}$ peptide (for example, $L_{11}$ for $L_{5} R L_{5}$ ). The difference between the experimental (blue) and simulation (red) $\Delta \Delta G$ is almost entirely due to the constant free-energy offset of the polyleucine peptides, with a small contribution due to differing slopes of the arginine-containing peptides. Due to deeper burial of the guanidinium side chain for longer peptides, the penalty of swapping a leucine with an arginine at the center of a polyleucine segment increases linearly with peptide length (see Fig. 3; Supplementary Table 2).

higher number of transitions between states are more reliable. Because the rate of transitions between states decline exponentially with increasing free-energy differences, the $\mathrm{L}_{6} \mathrm{RL}_{6}$ and $\mathrm{L}_{7} \mathrm{RL}_{7}$ peptides have the most reliable free energies (Supplementary Fig. 10).

Faster convergence of the thermodynamic equilibrium was achieved when the simulation temperature was elevated to $140^{\circ} \mathrm{C}$. We have previously shown that such high temperatures do not affect the thermodynamic equilibrium, because TM helices are highly stable against thermal denaturation due to the large penalty associated with breaking a peptide hydrogen bond in the hydrophobic membrane interior ${ }^{13}$ and the decreased aqueous solubility of hydrophobic molecules at elevated temperatures. Consequently, the conformational ensemble of the peptide does not change measurably with temperature. We experimentally verified the thermostability of the peptides embedded in POPC vesicles using SRCD spectroscopy, with essentially no unfolding detected at temperatures up to $85^{\circ} \mathrm{C}$ (see Supplementary Fig. 11). To verify that the partitioning behaves similarly above the boiling point, we carried out a 6- $\mu$ s control simulation of $\mathrm{L}_{6} \mathrm{RL}_{6}$ at $90^{\circ} \mathrm{C}$. The results show that the insertion propensity $P_{\mathrm{TM}}=29.9 \pm 24.4$ (s.d.) at $90^{\circ} \mathrm{C}$ is similar to the $140{ }^{\circ} \mathrm{C}$ system $\left(P_{\mathrm{TM}}=35.3 \pm 7.6\right.$ 
(s.d.)) (see Table 1). However, the much smaller number of transitions at the lower temperature resulted in large errors and poor convergence (see Supplementary Fig. 10). We estimate that even at $90^{\circ} \mathrm{C}$ the simulation would have to be extended by roughly an order of magnitude to achieve the same level of convergence as the $140-{ }^{\circ} \mathrm{C}$ system.

\section{Discussion}

Figure 4 compares the propensity $P_{\mathrm{TM}}$ and free energy $\Delta G_{\mathrm{TM}}$ of TM insertion obtained from the simulations and experiments. Although the OSRCD measurements could only provide estimates of the orientation state population for $\mathrm{L}_{6} \mathrm{RL}_{6}$ and $\mathrm{L}_{7} \mathrm{RL}_{7}$, the general agreement between all methods is remarkable. The results suggest that 14 or more leucine residues are sufficient for favourable TM insertion of a hydrophobic polyleucine segment containing a central arginine.

We have previously determined the transfer free energies of polyleucine peptides using both folding-partitioning simulations as well as the translocon assay described above ${ }^{13,14}$. The contribution of the added arginine side chain to the transfer free energies of the peptides can be estimated by subtracting the transfer free energy of the corresponding polyleucine segments. The analysis reveals that replacing a leucine with an arginine residue at the center of a polyleucine segment results in a freeenergy penalty that rises linearly with the length of the peptide (Fig. 4b; Supplementary Table 2). The linear rise occurs because longer peptides result in deeper burial of the guanidinium group (Supplementary Fig. 6). For translocon partitioning, the freeenergy penalty rises from $2.4 \mathrm{kcal} \mathrm{mol}^{-1}$ for replacing the central leucine of $\mathrm{L}_{11}$ with an arginine, to $4.3 \mathrm{kcalmol}^{-1}$ for $\mathrm{L}_{17}$.
Swapping the central leucine with an arginine is more costly in the simulations, with a penalty of $5.4 \mathrm{kcal} \mathrm{mol}^{-1}$ for $\mathrm{L}_{11}$ and $6.8 \mathrm{kcal} \mathrm{mol}^{-1}$ for $\mathrm{L}_{17}$. The discrepancy between the experimental and simulation data seems exclusively due to the constant offset of $2 \mathrm{kcal} \mathrm{mol}^{-1}$ observed between simulation- and transloconderived insertion free energies of pure polyleucine peptides (Supplementary Table 2).

Overall, the free energy penalty for burial of a charged guanidinium group at the heart of a hydrophobic membranespanning segment is moderate and consistent with earlier translocon data, which measured only minor energetic penalties $^{9,10}$. The present approach suggests that using simulations to provide an atomic-detail picture of the system can provide complementary insights for experimental measurements that are not possible to achieve with either approach individually. In the present case, the experimental OSRCD and translocon measurements provide time-averaged structural and energetic equilibrium data, while the simulations report the peptide configurations and dynamics in the bilayer that give rise to these thermodynamic measurements. This knowledge of the equilibrium states, between which the equilibrium is measured, is vital for the development of accurate models.

The reasons for the offset of the polyleucine partitioning free energies, especially in light of the excellent agreement between translocon and simulation data for the arginine-containing peptides presented here, are currently not clear. One interpretation might be that a charge inside the hydrophobic segment might give the translocon less leeway than a pure hydrophobic segment. It has been suggested that arginine insertion into bilayers is favourable due to dimerization ${ }^{25}$. Although the simulation data show that the peptides insert efficiently as

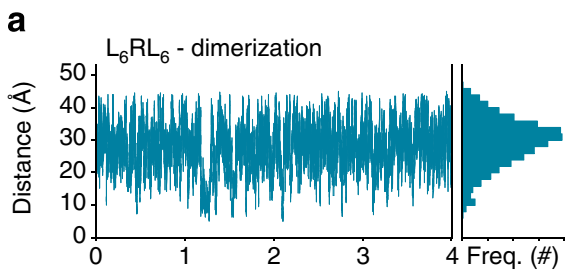

d
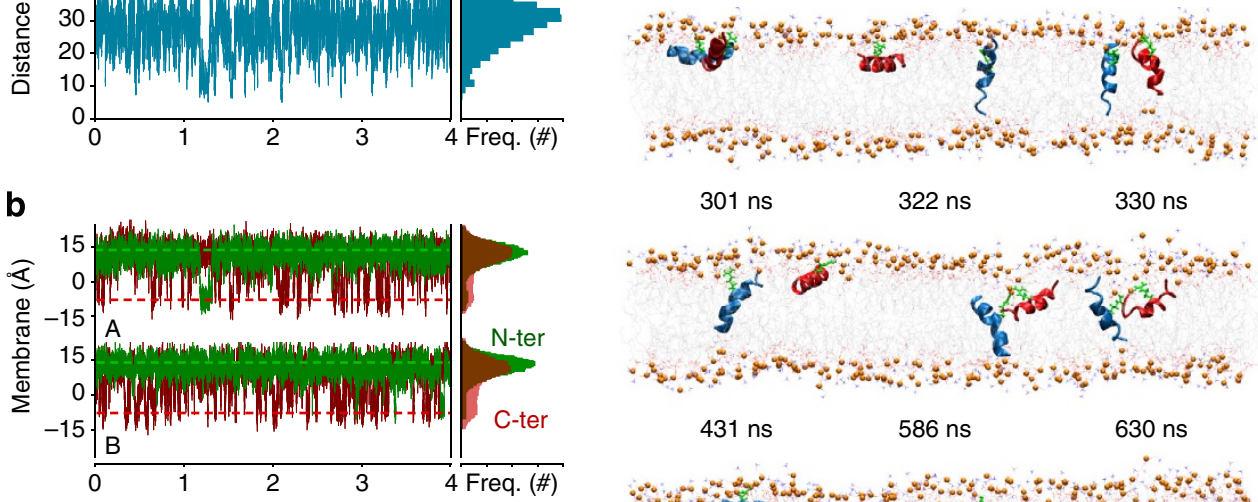

$301 \mathrm{~ns} \quad 322 \mathrm{~ns} \quad 330 \mathrm{~ns}$
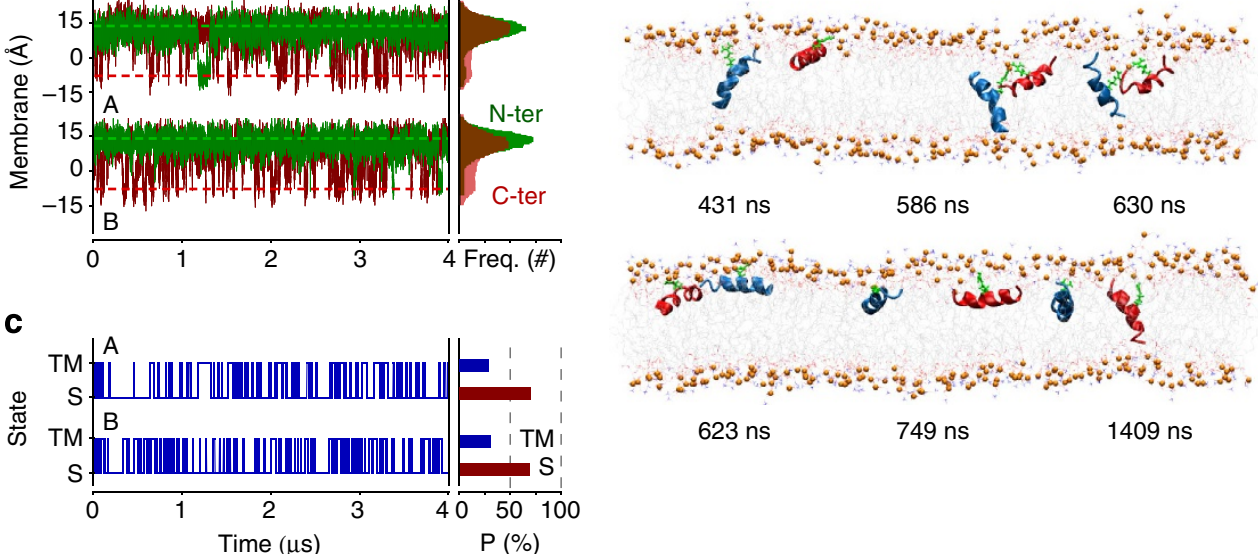

$623 \mathrm{~ns}$

749 ns

1409 ns

Figure 5 | Equilibrium simulation $(\mathbf{4} \boldsymbol{\mu} \mathbf{s})$ of two $\mathbf{L}_{\mathbf{6}} \mathbf{R L}_{\mathbf{6}}$ peptides to test for dimerization. (a) Analysis of the inter-arginine distance. The figure plots the distance between the $\mathrm{CZ}$ atoms of the two guanidinium side chains. The figure shows that the peptides diffuse randomly, independent of each other and any aggregate formation is short-lived, with the longest observed contact lasting $\sim 90 \mathrm{~ns}$ (at $1.17 \mu \mathrm{s}$ ). (b) Analysis of the positions of the peptide $\mathrm{N}$ termini (green) and $\mathrm{C}$ termini (red) along the membrane normal reveals that each peptide partitions independently of the other. (c) The state transitions from the analysis of the peptide termini position along the membrane normal (b) show that both peptides have similar S- and TM-state populations and associated free energies (see Table 1). (d) Snapshots of the dimer simulation show independent partitioning of the peptides with occasional random collision, no stable aggregate formation was observed. See movies LnRLn_dimer_side.mov and LnRLn_dimer_top.mov in online Supplementary Information. 
monomers, this does not preclude a further lowering of the insertion free energy for dimeric TM configurations, which might reintroduce the offset between translocon and simulation data observed for pure polyleucine peptides.

To test the dimerization hypothesis, we performed a simulation with two $\mathrm{L}_{6} \mathrm{RL}_{6}$ peptides in the same bilayer patch. The results, summarized in Fig. 5, show that although the peptides meet many times during the course of the simulation, and occasionally form a common defect in the membrane, aggregates have a short lifetime. We conclude that the two peptides generally partition independently of each other, particularly because the insertion propensity $P_{\mathrm{TM}}=30.2 \pm 7.9 \%$ is similar for both peptides and close to the value observed for the single peptide simulation $(35.3 \pm 7.6 \%)$. Furthermore, the two-peptide data suggest that partitioning of the peptides is monomeric, with no further energetic gain through aggregate formation, although it should be noted that the timescale of the simulations might be too short to capture the formation of a stable aggregate.

A particularly important observation is that during the simulations the initially unfolded peptides in solution quickly partition into the lipid bilayer interface, never to leave, so that the equilibrium states observed are interfacial and TM. This means that the free energies measured in the simulation are those for transferring the helices from the bilayer interface into and across the bilayer (Fig. 1b). The interfacial location is expected from extensive experimental studies of the partitioning of amphipathic peptides, such as melittin ${ }^{37-40}$. A folded interfacial state is preferred because of the high cost of partitioning peptide bonds into the interface or across the bilayer ${ }^{2}$. The formation of intramolecular hydrogen bonds of $\alpha$-helices markedly reduces the cost of partitioning, making the folded interfacial state the preferred state, as observed here. There is no reason to believe that interfacial folding is not also important biologically. Unless the translocon completely isolates nascent helices emerging from the ribosome, helix-interface interactions are likely to be important in translocon-guided insertion. In fact, we know that the translocon-ribosome connection is quite exposed to the cytoplasm $^{41-44}$. One might thus speculate that the membrane interface in the vicinity of the translocon plays an important role in translocon-guided TM helix insertion.

\section{Methods}

Peptide designs. Translocon experiments. The flanks of the $\mathrm{L}_{n} \mathrm{RL}_{n}$ hydrophobic segment were extended to include-GGPG- at the N-terminal end and -GPGG- at the C-terminal end to insulate the hydrophobic segment from the surrounding LepB carrier protein.

Synthetic experiments. Acetyl-GWL $\mathrm{GW}_{n-1}-\mathrm{R}-\mathrm{L}_{n} \mathrm{G}$-amide $(n=5-8)$. The $\mathrm{N}$-terminal leucine of each peptide was replaced with a tryptophan to aid purification and quantification of peptide concentration for the spectroscopic experiments, exploiting the average molar extinction coefficient of $\varepsilon=5,600 \mathrm{M}^{-1} \mathrm{~cm}^{-1}$ of the tryptophan indole moiety at $280 \mathrm{~nm}^{45}$.

Computational experiments. Ace- $\mathrm{GL}_{n}-\mathrm{R}-\mathrm{L}_{n} \mathrm{G}$-ame $(n=5-8)$. The $\mathrm{N}$ - and $\mathrm{C}$ termini were acetylated (ace) and amidated (ame), respectively, to reduce the kinetic barrier associated with TM insertion of the peptide, which involves transport of a polar peptide terminus across the hydrophobic membrane core.

Sec61 translocon insertion assay. Enzymes and chemicals. Unless stated otherwise, all chemicals were from Sigma-Aldrich (St Louis, MO, USA). Oligonucleotides were purchased from MWG Biotech AG (Ebersberg, Germany). Pfu Turbo DNA polymerase was purchased from Agilent Technologies. All other enzymes were from Fermentas. The plasmid pGEM-1 and the TNT SP6 Transcription/ Translation System were from Promega. $\left[{ }^{35} \mathrm{~S}\right]$ Met was from PerkinElmer.

DNA manipulations. All LepB constructs used carried one acceptor site for $\mathrm{N}$-linked glycosylation in positions 97-99 (Asn-Ser-Thr) and the naturally present acceptor site in positions 215-217 (Asn-Glu-Thr). LepB constructs encoding $\mathrm{H}$-segments with different numbers of leucines were generated by modifying the lepB gene in the pGEM-1 vector containing SpeI and KpnI restriction sites? Double-stranded oligonucleotides encoding H-segments with GGPG/GPGG flanks including either $\mathrm{N}$-terminally a SpeI or C-terminally a KpnI restriction site were created by annealing two pairs of complementary oligonucleotides with overlapping overhangs. All sequences encoding different $\mathrm{H}$-segments and the pGEM-1 vector were digested with SpeI and KpnI, followed by insertion of the $\mathrm{H}$-segments into the cut vector by ligation. Oligonucleotides were diluted to a concentration of $20 \mu \mathrm{M}$. Twenty microlitres of each forward and reverse complementary segment (sample 1: forward $1+$ reverse 1 ; sample 2 : forward $2+$ reverse 2 ) were mixed with $4.4 \mu \mathrm{l}$ annealing oligonucleotide buffer (20 mM Tris- $\mathrm{HCl} \mathrm{pH} 7.4,20 \mathrm{mM} \mathrm{MgCl}_{2}$ and $500 \mathrm{mM} \mathrm{NaCl}$ ) and heated at $85^{\circ} \mathrm{C}$ for $10 \mathrm{~min}$. Samples were left in the heating block until the temperature was $<30^{\circ} \mathrm{C}$. Twenty microlitres each of the annealed samples 1 and 2 were mixed and heated at $65^{\circ} \mathrm{C}$ for $5 \mathrm{~min}$. Samples were left in the heating block until the temperature was $<30^{\circ} \mathrm{C}$. The annealed $\mathrm{H}$-segment was ligated into the cut vector overnight at $16^{\circ} \mathrm{C}$ followed by transformation. All inserts and mutagenesis were confirmed by sequencing of plasmid DNA at Eurofins MWG Operon (Ebersberg, Germany). Oligonucleotide sequences were as follows:

$\mathrm{L}_{5} \mathrm{RL}_{5}$ : forward first part: $5^{\prime}$-P-CTAGTGGTGGTCCTGGActgcttctgcttctgcgtctg$3^{\prime}$; forward second part: $5^{\prime}$-P-cttctgcttctgGGACCTGGTGGGGTAC-3'; reverse first part: $5^{\prime}$-P-cagaagcagTCCAGGACCACCA-3'; reverse second part: $5^{\prime}$-P-CCCACC AGGTCCcagaagcagaagcagacgcagaag- $3^{\prime}$.

$\mathrm{L}_{6} \mathrm{RL}_{6}$ : forward first part: $5^{\prime}$-P-CTAGTGGTGGTCCTGGActgctgcttctgcttctgcgt ctg- $3^{\prime}$; forward second part: $5^{\prime}$-P-cttctgcttctgctgGGACCTGGTGGGGTAC-3'; reverse first part: $5^{\prime}$-P-cagaagcagcagTCCAGGACCACCA- $3^{\prime}$; reverse second part: 5'-P-CCCACCAGGTCCcagcagaagcagaagcagacgcagaag- $3^{\prime}$.

$\mathrm{L}_{7} \mathrm{RL}_{7}$ : forward first part: $5^{\prime}$-P-CTAGTGGTGGTCCTGGActgctgctgcttctgctt ctgcgtctg- $3^{\prime}$; forward second part: $5^{\prime}$-P-cttctgcttctgctgctgGGACCTGGTGGGG TAC- $3^{\prime}$; reverse first part: $5^{\prime}$-P-cagaagcagcagcagTCCAGGACCACCA- $3^{\prime}$; reverse second part: $5^{\prime}$-P-CCCACCAGGTCCcagcagcagaagcagaagcagacgcagaag- $3^{\prime}$.

$\mathrm{L}_{8} \mathrm{RL}_{8}$ : forward first part:5'-P-CTAGTGGTGGTCCTGGActgctgctgctgcttctgctt ctgcgtctg- $3^{\prime}$; forward second part: $5^{\prime}$-P-cttctgettctgctgctgctgGGACCTGGTGGGGT AC- $3^{\prime}$; reverse first part: $5^{\prime}$-P-cagaagcagcagcagcagTCCAGGACCACCA- $3^{\prime}$; reverse second part: $5^{\prime}$-P-CCCACCAGGTCCcagcagcagcagaagcagaagcagacgcagaag- $3^{\prime}$.

$\mathrm{L}_{9} \mathrm{RL}_{9}$ : forward first part: $5^{\prime}$-P-CTAGTGGTGGTCCTGGActgctgctgctgctgcttct gcttctgcgtctg- $3^{\prime}$; forward second part: $5^{\prime}$-P-cttctgcttctgctgctgctgctgGGACCTGGT GGGGTAC- $3^{\prime}$; reverse first part: $5^{\prime}$-P-cagaagcagcagcagcagcagTCCAGGACCA CCA- $3^{\prime}$; reverse second part: $5^{\prime}$-P-CCCACCAGGTCCcagcagcagcagcagaagcagaagca gacgcagaag- $3^{\prime}$.

Expression in vitro. Constructs cloned in pGEM-1 were transcribed and translated in the TNT Quick coupled transcription/translation system. One microgram of DNA template, $1 \mu \mathrm{l}$ of $\left[{ }^{35} \mathrm{~S}\right] \mathrm{Met}(10 \mu \mathrm{Ci} ; 1 \mathrm{Ci}=37 \mathrm{GBq}), 1 \mu \mathrm{l}$ of RNasin, $1 \mu \mathrm{l}$ of a mixture of all 20 natural amino acids minus methionine and $1 \mu \mathrm{l}$ of dog pancreas RMs were mixed with $9 \mu$ l of TNT lysate mix, and samples were incubated for $90 \mathrm{~min}$ at $30^{\circ} \mathrm{C}$. The sample was mixed with SDS sample buffer ${ }^{10}$ and incubated at $90^{\circ} \mathrm{C}$ for $5 \mathrm{~min}$ before loading on a $12 \%$ SDS/polyacrylamide gel. Proteins bands were visualized in a Fuji FLA-3000 phosphoimager (Fujifilm, Tokyo, Japan). The Image Gauge V 4.23 software (Fujifilm) was used to generate a two-dimensional intensity profile of each gel lane, and the multi-Gaussian fit program from the Qtiplot software package (www.qtiplot.ro) was used to calculate the peak areas of the protein bands. An apparent equilibrium constant between the membrane-integrated and non-integrated forms was calculated as: $K_{\text {app }}=f_{1 \mathrm{~g}} / f_{2 \mathrm{~g}}$, where $f_{1 \mathrm{~g}}$ is the fraction of singly glycosylated LepB molecules and $f_{2 \mathrm{~g}}$ is the fraction of doubly glycosylated LepB molecules. The results were then converted to apparent free energies, $\Delta G_{\text {app }}=-R T \ln K_{\text {app }}$.

Synthetic peptide experiments. Synthesis and purification. All peptides were solid-state synthesized on amide resins using standard FMOC/HBTU chemistry, purified using reverse-phase high-pressure liquid chromatography, and quantified using mass spectroscopy. The peptides were injected in $100 \%$ formic acid and separated using C4-C18 columns with acetonitrile-water gradients $(0.1 \%$ trifluoroacetic acid) and lyophilized.

Sample preparation. Samples were prepared by mixing stock solutions of peptide in trifluorethanol and POPC lipids in chloroform (Avanti Polar Lipids, Alabaster, AL, USA). The mixture was desolvated under a stream of nitrogen with subsequent overnight incubation under vacuum to remove solvent. Vesicles were prepared by rehydrating dry peptide/lipid films in distilled water at room temperature. Vesicles were prepared by extrusion through membranes with $200-\mathrm{nm}$ pores. The peptide concentration in all samples was $30 \mu \mathrm{M}$, and the molar peptide/lipid ratio was $1 / 100$. Stacked lipid bilayers were prepared by dissolving peptide/lipid mixtures in trifluorethanol and application to Suprasil quartz slides at peptide/lipid ratios of $1 / 20-1 / 200$, and hydrating them in a specially designed sample chamber ${ }^{46}$. The peptide concentrations of all samples were $1 \mathrm{mg} \mathrm{ml}^{-1}$.

SRCD spectroscopy. Circular dichroism spectra were collected at the CD12 beamline at ANKA (Karlsruhe, Germany), the CD1 beamline at ISA (Århus, Denmark), and beamline 4B8 at the Beijing Synchrotron Radiation Facility (China $)^{47}$. OSRCD spectra were collected for eight rotation angles of the quartz substrate around the axis of the beam and averaged to limit linear dichroism effects. Data were collected from 280 to $180 \mathrm{~nm}$ at $1 \mathrm{~nm}$ intervals and $1 \mathrm{~nm}$ bandwidth. The response time was $2-4 \mathrm{~s}$ at each wavelength and each measurement was an average of four scans. Temperature was controlled with a Peltier device, and varied between 20 and $85^{\circ} \mathrm{C}$ in $5^{\circ} \mathrm{C}$ steps. Samples were equilibrated for at least $5 \mathrm{~min}$ at each temperature before data collection. All spectra were corrected for background by subtracting the corresponding vesicle-only spectrum measured over 
the same temperature range. Spectral and metadata deposited in the Protein Circular Dichroism Data Bank will be released upon publication.

Molecular dynamics simulations. Simulations were performed and analysed with Gromacs 4.5 (www.gromacs.org) ${ }^{48}$ and hippo beta (www.biowerkzeug.com), using the OPLS all-atom protein force field ${ }^{49}$ in conjunction with the TIP3P water model $^{50}$. OPLS united-atom lipid parameters for POPC were taken from Ulmschneider et al. ${ }^{35}$ Control simulations were carried out with the CHARMM27 protein, and CHARMM36 lipid force fields, with TIP3P water. Electrostatic interactions were computed using Particle mesh Ewald (PME) algorithm. A cutoff of $10 \AA$ was used for van der Waals interactions. Bonds involving hydrogen atoms were constrained using LINCS ${ }^{51}$. The integration time-step was $2 \mathrm{fs}$ and neighbour lists were updated every five steps. All simulations were performed in the isothermal isobaric ensemble with constant particle number (NpT), without any restraints or biasing potentials. Water, lipids and the protein were each coupled separately to a heat bath with time constant $\tau_{\mathrm{T}}=0.1 \mathrm{ps}$ using weak temperature coupling $^{52}$. Atmospheric pressure of 1 bar was maintained ${ }^{53}$ using weak semiisotropic pressure coupling with compressibility $\kappa_{z}=\kappa_{x y}=4.6 \times 10^{-5}$ bar $^{-1}$ and time constant $\tau_{\mathrm{P}}=1$ ps. Before production runs all systems were equilibrated for $2-10 \mathrm{~ns}$.

Free-energy surfaces. The free-energy surfaces were calculated as a function of the peptide's center-of-mass position along the membrane normal and the tilt angle. A two-dimensional normalized histogram was calculated over the equilibrium phase of the simulations. Each 'bin' contains the probability $p_{\mathrm{i}}$ of finding the peptide in state i. For a system in thermodynamic equilibrium, the change in free energy on going from one state to another is given by

$$
\Delta G=-R T \ln \frac{p_{1}}{p_{2}}
$$

where $R$ is the ideal gas constant and $T$ is the temperature. The relative free energy was calculated for each bin, and the minimum was shifted to zero.

Insertion propensity and free energy. The insertion propensity $P_{\mathrm{TM}}=f_{\mathrm{TM}} /\left(f_{\mathrm{TM}}+f_{\mathrm{S}}\right)$ of each peptide is calculated as the probability of the peptide being in the TM state. To distinguish the TM state from the $\mathrm{S}$ conformations, the position of the peptide termini along the membrane normal was tracked.

The insertion free energy, or 'free energy of partitioning' can be directly calculated from the TM-state propensity using

$$
\Delta G_{\mathrm{S} \rightarrow \mathrm{TM}}=-R T \ln \left(1 / P_{\mathrm{TM}}-1\right)
$$

Helicity. The helicities of the peptides were calculated for each residue, and then averaged over the complete sequence. A residue is considered helical if its backbone dihedral angles are within $\pm 40^{\circ}$ from the ideal $\alpha$-helical values $\left(\Phi=-57^{\circ}, \Psi=-47^{\circ}\right)$

Error estimates. In all figures, we determined error bar estimates by dividing the simulations into a series of 10 blocks of equal length, and then performing averaging for each block. The error bars are given by $\pm \sigma$, where $\sigma$ is the s.d. of the mean, that is, the s.d. of the individual block averages with respect to the overall average.

\section{References}

1. Engelman, D. M., Steitz, T. A. \& Goldman, A. Identifying nonpolar transbilayer helices in amino acid sequences of membrane proteins. Annu. Rev. Biophys. Biophys. Chem. 15, 321-353 (1986).

2. White, S. H. \& Wimley, W. C. Membrane protein folding and stability: Physical principles. Annu. Rev. Biophys. Biomol. Struct. 28, 319-365 (1999).

3. Kyte, J. \& Doolittle, R. F. A simple method for displaying the hydropathic character of a protein. J. Mol. Biol. 157, 105-132 (1982).

4. Jayasinghe, S., Hristova, K. \& White, S. H. Energetics, stability, and prediction of transmembrane helices. J. Mol. Biol. 312, $927-934$ (2001).

5. Osborne, A. R., Rapoport, T. A. \& Van den Berg, B. Protein translocation by the Sec61/SecY channel. Annu. Rev. Cell Dev. Biol. 21, 529-552 (2005).

6. White, S. H. \& von Heijne, G. Transmembrane helices before, during, and after insertion. Curr. Opin. Struct. Biol. 15, 378-386 (2005).

7. Park, E. \& Rapoport, T. Mechanisms of Sec61/SecY-mediated protein translocation across membranes. Annu. Rev. Biophys. 41, 21-40 (2012).

8. Dalbey, R. E., Wang, P. \& Kuhn, A. Assembly of bacterial inner membrane proteins. Annu. Rev. Biochem. 80, 161-187 (2011).

9. Hessa, T. et al. Recognition of transmembrane helices by the endoplasmic reticulum translocon. Nature 433, 377-381 (2005).

10. Hessa, T. et al. The molecular code for transmembrane-helix recognition by the Sec61 translocon. Nature 450, 1026-1030 (2007).

11. White, S. H. \& Von Heijne, G. How translocons select transmembrane helices. Annu. Rev. Biophys. 37, 23-42 (2008).
12. Wimley, W. C. \& White, S. H. Designing transmembrane a-helices that insert spontaneously. Biochemistry 39, 4432-4442 (2000).

13. Ulmschneider, J. P., Smith, J. C., White, S. H. \& Ulmschneider, M. B. In silico partitioning and transmembrane insertion of hydrophobic peptides under equilibrium conditions. J. Am. Chem. Soc. 133, 15487-15495 (2011).

14. Jaud, S. et al. Insertion of short transmembrane helices by the Sec61 translocon. Proc. Natl Acad. Sci. USA 106, 11588-11593 (2009).

15. Gleason, N. J., Vostrikov, V. V., Greathouse, D. V. \& Koeppe, II R. E. Buried lysine, but not arginine, titrates and alters transmembrane helix tilt. Proc. Natl Acad. Sci. USA 110, 1692-1695 (2013).

16. Li, L., Vorobyov, I., MacKerell, Jr. A. D. \& Allen, T. J. Is arginine charged in a membrane? Biophys. J. 94, L11-L13 (2008).

17. Dorairaj, S. \& Allen, T. W. On the thermodynamic stability of a charged arginine side chain in a transmembrane helix. Proc. Natl Acad. Sci. USA 104, 4943-4948 (2007).

18. Vorobyov, I., Li, L. \& Allen, T. W. Assessing atomistic and coarse-grained force fields for protein-lipid interactions: the formidable challenge of an ionizable side chain in a membrane. J. Phys. Chem. 112, 9588-9602 (2008).

19. Johansson, A. C. V. \& Lindahl, E. Position-resolved free energy of solvation for amino acids in lipid membranes from molecular dynamics simulations. Proteins 70, 1332-1344 (2008).

20. Johansson, A. C. \& Lindahl, E. The role of lipid composition for insertion and stabilization of amino acids in membranes. J. Chem. Phys. 130, 185101 (2009).

21. Johansson, A. C. V. \& Lindahl, E. Titratable amino acid solvation in lipid membranes as a function of protonation state. J. Phys. Chem. 113, 245-253 (2009).

22. MacCallum, J. L., Bennett, W. F. D. \& Tieleman, D. P. Partitioning of amino acid side chains into lipid bilayers: Results from computer simulations and comparison to experiment. J. Gen. Physiol. 129, 371-377 (2007).

23. MacCallum, J. L., Bennett, W. F. D. \& Tieleman, D. P. Distribution of amino acids in a lipid bilayer from computer simulations. Biophys. J. 94, 3393-3404 (2008).

24. Schow, E. V. et al. Arginine in membranes: the connection between molecular dynamics simulations and translocon-mediated insertion experiments. $J$. Membr. Biol. 239, 35-48 (2011).

25. Rychkova, A., Vicatos, S. \& Warshel, A. On the energetics of transloconassisted insertion of charged transmembrane helices into membranes. Proc. Natl Acad. Sci. USA 107, 17598-17603 (2010).

26. Rychkova, A. \& Warshel, A. On the nature of the apparent free energy of inserting amino acids into membrane through the translocon. J. Phys. Chem. B 117, 13748-13754 (2013).

27. Gumbart, J., Chipot, C. \& Schulten, K. Free-energy cost for translocon-assisted insertion of membrane proteins. Proc. Natl Acad. Sci. USA 108, 3596-3601 (2011).

28. Hristova, K. et al. An amphipathic a-helix at a membrane interface: a structural study using a novel x-ray diffraction method. J. Mol. Biol. 290, 99-117 (1999).

29. Hristova, K., Dempsey, C. E. \& White, S. H. Structure, location, and lipid perturbations of melittin at the membrane interface. Biophys. J. 80, 801-811 (2001).

30. Krepkiy, D. et al. Structure and hydration of membranes embedded with voltage-sensing domains. Nature 462, 473-479 (2009).

31. Vogel, H. Comparison of the conformation and orientation of alamethicin and melittin in lipid membranes. Biochemistry 26, 4562-4572 (1987).

32. Wu, Y., Huang, H. W. \& Olah, G. A. Method of oriented circular dichroism. Biophys. J. 57, 797-806 (1990).

33. Ulmschneider, M. B., Doux, J. P. F., Killian, J. A., Smith, J. \& Ulmschneider, J. P. Mechanism and kinetics of peptide partitioning into membranes. J. Am. Chem. Soc. 132, 3452-3460 (2010).

34. Ulmschneider, M. B., Smith, J. C. \& Ulmschneider, J. P. Peptide partitioning properties from direct insertion studies. Biophys. J. 98, L60-L62 (2010).

35. Ulmschneider, J. P. \& Ulmschneider, M. B. United atom lipid parameters for combination with the optimized potentials for liquid simulations all-atom force fiels. J. Chem. Theory Comput. 5, 1803-1813 (2009).

36. Klauda, J. B. et al. Update of the CHARMM all-atom additive force field for lipids: validation on six lipid types. J. Phys. Chem. B 114, 7830-7843 (2010).

37. Wimley, W. C. et al. Folding of $\beta$-sheet membrane proteins: A hydrophobic hexapeptide model. J. Mol. Biol. 277, 1091-1110 (1998).

38. Ladokhin, A. S. \& White, S. H. Folding of amphipathic $\alpha$-helices on membranes: energetics of helix formation by melittin. J. Mol. Biol. 285, 1363-1369 (1999).

39. Fernández-Vidal, M., Jayasinghe, S., Ladokhin, A. S. \& White, S. H. Folding amphipathic helices into membranes: amphiphilicity trumps hydrophobicity. J. Mol. Biol. 370, 459-470 (2007).

40. Almeida, P. F., Ladokhin, A. S. \& White, S. H. Hydrogen-bond energetics drive helix formation in membrane interfaces. Biochim. Biophys. Acta 1818, 178-182 (2012).

41. Ménétret, J.-F. et al. Single copies of Sec61 and TRAP associate with a nontranslating mammalian ribosome. Structure 16, 1126-1137 (2008). 
42. Cheng, Z. \& Gilmore, R. Slow translocon gating causes cytosolic exposure of transmembrane and lumenal domains during membrane protein integration. Nat. Struct. Mol. Biol. 13, 930-936 (2006).

43. Frauenfeld, J. et al. Cryo-EM structure of the ribosome-SecYE complex in the membrane environment. Nat. Struct. Mol. Biol. 18, 614-621 (2011).

44. Pfeffer, S. et al. Structure and 3D arrangement of endoplasmic reticulum membrane-associated ribosomes. Structure 20, 1508-1518 (2012).

45. Pace, C. N., Vajdos, F., Fee, L., Grimsley, G. \& Gray, T. How to measure and predict the molar absorption coefficient of a protein. Protein Sci. 4, 2411-2423 (1995).

46. Wien, F. \& Wallace, B.A. Calcium fluoride micro cells for synchrotron radiation circular dichroism spectroscopy. Appl. Spectroscopy 59, 1109-1113 (2005).

47. Wallace, B.A. Protein characterisation by synchrotron radiation circular dichroism (SRCD) spectroscopy. Quart. Rev.Biophys 42, 317-370 (2009).

48. Berendsen, H. J. C., van der Spoel, D. \& van Drunen, R. GROMACS: a new message-passing parallel molecular dynamics implemetation. Comput. Phys. Commun. 91, 43-56 (1995).

49. Jorgensen, W. L., Maxwell, D. S. \& Tirado-Rives, J. Development and testing of the OPLS all-atom force field on conformational energetics and properties of organic liquids. J. Am. Chem. Soc. 118, 11225-11236 (1996).

50. Jorgensen, W. L., Chandrasekhar, J., Madura, J. D., Impey, R. W. \& Klein, M. L. Comparison of simple potential functions for simulating liquid water. J. Chem. Phys. 79, 926-935 (1983).

51. Hess, B., Bekker, H., Berendsen, H. J. C. \& Fraaije, J. G. E. M. LINCS: a linear constraint solver for molecular simulations. J. Comput. Biol. 18, 1463-1472 (1997).

52. Berendsen, H. J. C., Postma, J. P. M., van Gunsteren, W. F., DiNola, A. \& Haak, J. R. Molecular dynamics with coupling to an external bath. J. Chem. Phys. 81, 3684-3690 (1984)

53. Bussi, G., Donadio, D. \& Parrinello, M. Canonical sampling through velocity rescaling. J. Chem. Phys. 126, 014101 (2007).

\section{Acknowledgements}

The research was supported in part by NIH grants RO1 GM74637 and PO1 GM86685 to S.H.W.; an International Exchange Grant from the Royal Society (to B.A.W.); a grant from the National Natural Science Foundation of China (BC4190004) and a 'Young 1,000 Talent' grant to J.P.U.; by grants from the Swedish Cancer Foundation, the Swedish Research Council, the Swedish Foundation for Strategic Research, the European Research Council (ERC-2008-AdG 232648) and the Knut and Alice Wallenberg Foundation to G.v.H.; and by grants from the UK BBSRC to B.A.W. M.B.U. was supported at the early stages of the project by a Marie Curie Senior Fellowship. SRCD beam time was supported by grants from the ISA, ANKA and BSRF (China) synchrotrons to B.A.W.

\section{Author contributions}

M.B.U., G.v.H., and S.H.W. conceived the experiments. N.S. performed the translocon experiments. The experiments with and analyses of synthetic peptides were performed by M.B.U. and B.A.W. M.B.U. and J.P.U. carried out the MD simulations. M.B.U., G.v.H., and S.H.W. prepared the manuscript with discussions and improvements from all authors.

\section{Additional information}

Supplementary Information accompanies this paper at http://www.nature.com/ naturecommunications

Competing financial interests: The authors declare no competing financial interests.

Reprints and permission information is available online at http://npg.nature.com/ reprintsandpermissions/

How to cite this article: Ulmschneider, M. B. et al. Spontaneous transmembrane helix insertion thermodynamically mimics translocon-guided insertion. Nat. Commun. 5:4863 doi: 10.1038/ncomms5863 (2014). 